\title{
3D Modeling and Displaying System for Volume Communication*
}

\author{
Naohisa SAKAMOTO**, Nobuyuki KUKIMOTO***, Yukio YASUHARA****, \\ Yasuo EBARA $^{\dagger}$ and Koji KOYAMADA ${ }^{\dagger \dagger}$
}

\begin{abstract}
We developed a 3D modeling and displaying system for volume communication, which consists of a set of cameras and a PC cluster, and evaluated on both LCD and omni-directional displays. In this paper, we propose a parallel voxel coloring method for accelerating the 3D modeling process. By using a system consisting of five cameras and a six-node PC cluster, it was possible to model and display a real-world object at interactive frame rate. We also investigated the use of an omni-directional display and verified the effectiveness of sharing the same reconstructed object, showing the potential to become an important tool for volume communication.
\end{abstract}

Key Words: Volume Communication, Omni-Directional Display, 3D Reconstruction, Parallel Voxel Coloring

\section{Introduction}

In recent year, much research on tele-immersion technique has been developed because of the rapid advance on computer performance and network bandwidth as well as on graphics hardware acceleration techniques. Teleimmersion techniques enable users to share a virtual environment while achieving a high degree of reality between remote places $^{(1),(2)}$. In order to realize a tele-immersive environment, it is important to see the user images from remote places without any restriction about viewpoint in real-time. One of the most popular methods to obtain a user's viewpoint position is to use a special hardware device, such as an optical head tracker. In a traditional teleimmersive environment, a user is positioned in front of a face-to-face display, and multi-viewpoint video images

* Received 22nd November, 2004 (No. 04-4223)

** Graduate School of Engineering, Kyoto University, Yoshida-Honmachi, Sakyo, Kyoto 606-8501, Japan

*** Department of Information, Tohwa University, 1-1-1 Chikushigaoka, Minami, Fukuoka 815-8510, Japan

**** Faculty of Engineering, Kyoto University, YoshidaHonmachi, Sakyo, Kyoto 606-8501, Japan

$\dagger$ Academic Center for Computing and Media Studies, Kyoto University, Yoshida-Honmachi, Sakyo, Kyoto 6068501, Japan

† Center for the Promotion of Excellence in Higher Education, Kyoto University, Yoshida-Nihonmatsu, Sakyo, Kyoto 606-8501, Japan are captured by using multiple cameras. Moreover, these multi-viewpoint images are merged into a virtual space within a display system and displayed without sense of incompatibility.

In recent year, practical 3D display systems without need for any special glasses have been developed as a result of the advances in 3D display technology ${ }^{(3),(4)}$. By using this kind of 3D display system, many users can display the 3D image for each viewpoint at the same time. Therefore, we think that these technologies are effective for constructing a tele-immersive environment. As a result, by generating multi-viewpoint 3D images tailored for these display systems in advance, multiple users can share them within a tele-immersive environment at the same time. Furthermore, by using such a 3D display system without the use of positioning sensors or special glasses, which interfere with non-verbal communication, we may build a tele-immersive environment. In particular, an omnidirectional display system ${ }^{(5)}$ that multiple users can see around from different viewpoints a 3D image at the same time is considered as a new communication technique for collaborative work in tele-immersive environment.

In this paper, we discuss a 3D modeling and displaying system which enables to display not only 3D CG images generated from experimental and instrumental data but also 3D real image generated by using a 3D modeling system with multi-viewpoint cameras. In addition, our proposed system makes it possible to observe real scene in remote places, by connecting the $3 \mathrm{D}$ modeling system 


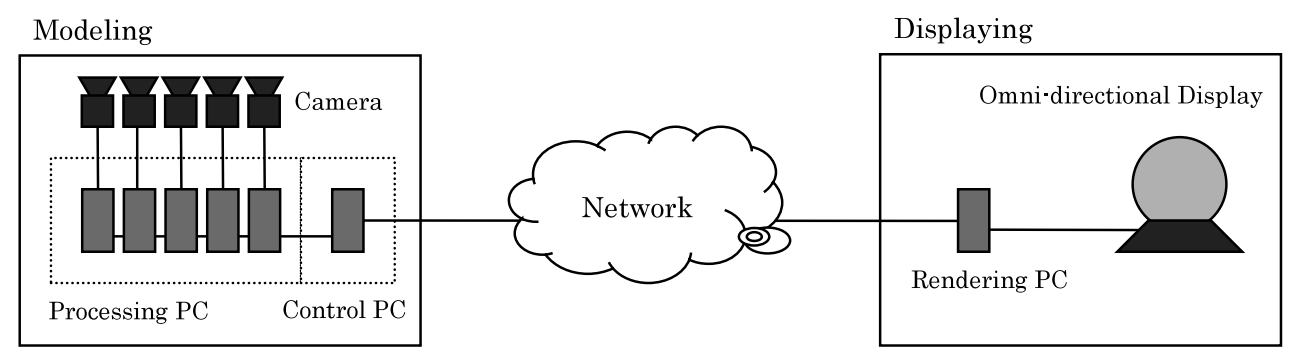

Fig. 1 System configuration

to a 3D display system using the omni-directional display via network.

\section{3D Reconstruction}

Much research on 3D shape reconstruction methods from multi-viewpoint images in the Computer Vision field has been done. In the case of using a face-to-face display in a tele-immersive environment, it is not absolutely necessary to reconstruct the complete object shape. In this case, some free-viewpoint image generation techniques $^{(6),(7)}$ can be applied.

On the other hand, in the case of using the aforementioned omni-directional display, it is necessary to reconstruct the shape of the object completely. In this case, the "shape from silhouette" technique, which can be performed stably without depending on the object's shape, is widely used. One of the most popular methods is the volume intersection method (VIM). In this method, the object shape is reconstructed based on view volume calculated from the silhouette image of the object. In addition, the voxel model of the object is generated by intersecting the view volumes calculated from each camera. Also, several acceleration techniques ${ }^{(8),(9)}$ have been proposed for this method. The most common approach for displaying a volume reconstructed by using VIM is to firstly convert the voxel model into a surface patch model and to then execute texture mapping on each patch. It is also possible to obtain a highly accurate reconstruction by adjusting the camera position and direction.

The shape data calculated from VIM is called "visual hull", and this calculated shape involves the true shape of the object which we are trying to obtain. Therefore, in order to obtain a shape closer to the true shape it is required to execute the fitting process on the surface patch model taking into account the silhouette images. This fitting process is computationally intensive and, as a result, it is not adequate for real-time processing.

To overcome this problem, Voxel Coloring method (VC) which avoids this conversion step has been proposed $^{(10)-(12)}$. The VC does not require the conversion step to the surface patch model and it assigns colors directly onto the voxels taking into consideration the captured images. In order to accurately perform the $\mathrm{VC}$, it is necessary to determine the visibility of voxels.
Culbertson et al. ${ }^{(13)}$ proposed the Generalized Voxel Coloring method (GVC) that eliminates its limitations from camera positioning. In this method, an item buffer is used to determine the visibility of the voxels. Prock et al. ${ }^{(14)}$ proposed an acceleration technique for the original $\mathrm{VC}$ method. It uses the octree structure to represent the volumetric field and a rough shape is reconstructed in the first step. Nowadays, there are remarkable advances in graphics hardware architecture. Taking advantage of this situation, several approaches that use graphics acceleration capability have been proposed ${ }^{(15)}$.

Taking into consideration that both the quantity of the cameras and the resolution of the volume field can become very large, it is easy to verify that a cluster of PCs is more appropriate than a single PC to support these scalability requirements. The $\mathrm{VC}$ enables processing in a voxel-byvoxel fashion and so is only required to parallelize the visibility determination stage in order to fully parallelize the VC. This makes it possible to run efficiently on PC clusters.

In this paper, we propose a parallel VC which uses the parallelization and the item buffer obtained from each camera in order to accelerate the voxel coloring process. To verify the effectiveness of our proposed method we developed a volumetric reconstruction system which enables image capturing, volume reconstruction and displaying. The hardware configuration used in the evaluation consists of a set of five cameras and a six-node PC cluster.

\section{System Configuration}

Our proposed system is composed of the modeling and displaying parts (Fig. 1).

\subsection{Modeling}

The 3D modeling part consists of two processes; (1) the initial voxel model generation process by using the parallel volume intersection method and (2) the colored voxel model generation process by using parallel voxel coloring method.

3.1.1 Parallel volume intersection An initial voxel model is generated by using the parallel volume intersection method from silhouette images generated using background subtraction. This process is as follows:

Step 1. The 3D space in which the object will be reconstructed is represented by a group of parallel planes. 
The silhouette image of each viewpoint is then projected onto the plane which will become the template for this group of parallel planes. The obtained result is called the base silhouette image.

Step 2. This base silhouette image is used to simulate actual projection onto a group of parallel planes by using the transformation and scaling alternately.

Step 3. The voxel data is generated by calculating the intersection of the projected silhouettes for each parallel plane from each viewpoint.

Step 4. Among the generated voxels, the voxels located in the surface of the object will be registered in a surface voxel list (SVL).

3.1.2 Parallel voxel coloring In order to perform voxel coloring accurately, the previous determination of the voxel's visibility becomes crucial. In our proposed method, the item buffer used in GVC is used for visibility determination. The main reason for choosing the item buffer is that these buffers can be generated for each camera independently from each other. As the cameras are each connected to a single PC the item buffer generation process is easily parallelized. There is a necessity to calculate the item buffers precisely. Although the item buffers are calculated by back-projection from the captured image, there is a possibility that errors are included in this visibility determination depending to the size of the projection image (footprint) of the voxel. To prevent such an error, accurate visibility determination based on footprint is required. In the following, we describe this process in detail.

Step 1. Back-projection of the eight vertices of a surface voxel.

Step 2. Footprint generation from projected voxel vertices.

Step 3. Determination of the distance between the camera and the voxel: This distance and the voxel ID (each voxel has your own unique ID) are assigned to the pixels within the generated footprint. However, in the case of an existing previous assignment, only the shortest distance is assigned to pixels.

The aforementioned steps are performed for all surface voxels within the volume data.

Step 4. Visibility determination of a given surface voxel is performed, checking whether all back-projected vertices are occluded.

By using the generated item buffers, we can determine the cameras from which a given surface voxel is visible. If a voxel is not visible from any camera, no action is performed, otherwise a color is assigned to this voxel.

During the color assigning process, color list for a given visible voxel is determined by back-projecting the central point of this voxel onto images selected in a previous stage. In order to obtain the final color, mean color value is calculated from the color list.
3.1.3 Implementation We have implemented the parallel voxel coloring method and we have run it on a PC cluster system. Our system consists of five processing PCs which we call "reconstruction node" and a control PC which we call "control node". These PCs are mutually connected by a Gigabit Ethernet interconnection network.

The proposed volumetric reconstruction process consists of the following three parts:

\section{A. Image Capturing \\ B. Voxel Model Generation \\ C. Colored Voxel Model Generation}

Each of the aforementioned parts is subdivided into a set of sub-processes (stages) and the entire process runs on each of the reconstruction nodes. Each reconstruction node has a single physical CPU. However, by activating the hyper-threading function we can treat them as though they have two logical CPUs. We made effective use of these resources and improved the system throughput by processing each stage in a pipeline. The implemented stages are as follows, and they are executed sequentially. The colored voxel model is finally generated in the control mode.
A. Image Capturing
1. Image Capturing (ICP) Stage
2. Image Correcting (ICR) Stage
B. Voxel Model Generation
3. Base Silhouette Image Generation (BSG) Stage
4. Slice Image Generation (SIG) Stage
5. Slice Image Distribution (SID) Stage
6. Voxel Model Generation (VMG) Stage
C. Colored Voxel Model Generation
7. Item-Buffer Generation (IBG) Stage
8. Item-Buffer Distribution (IBD) Stage
9. Colored Voxel Generation (CVG) Stage
10. Colored Voxel Distribution (CVD) Stage

\subsection{Displaying}

The displaying process is performed on the system that consists of a PC for rendering and the omnidirectional display, known as Perspecta spatial display developed by Actuality Systems Inc. ${ }^{(5)}$ (Fig. 2). This display is connected with the rendering PC by the SCSI- 2 cable.

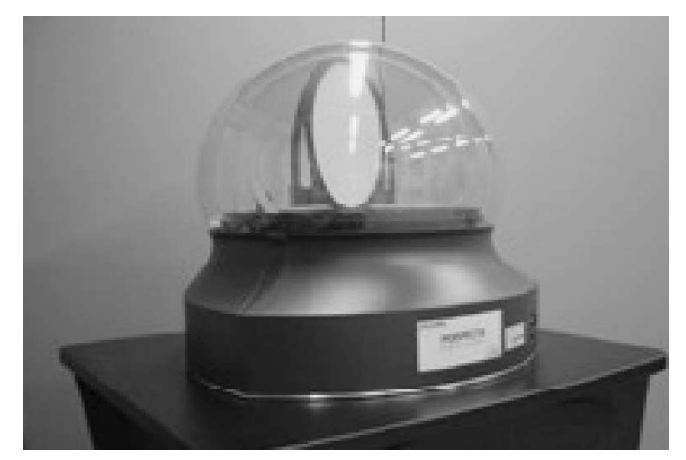

Fig. 2 Omni-directional Display (Perspecta) 

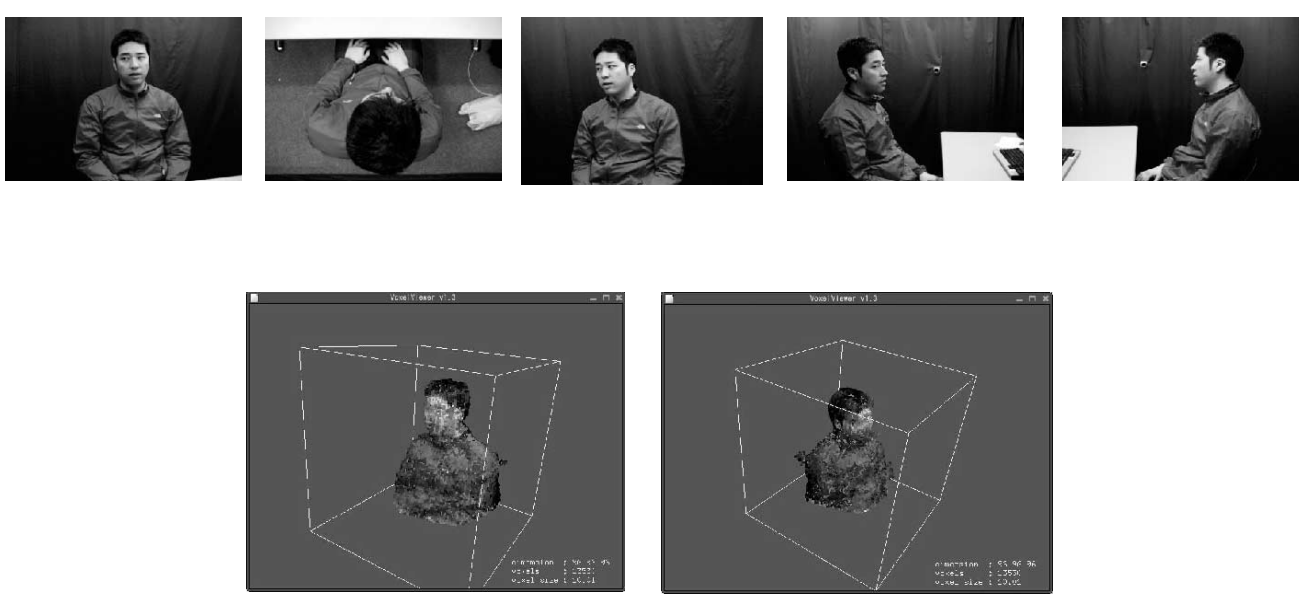

Fig. 4 Example of some captured images and reconstructed object images
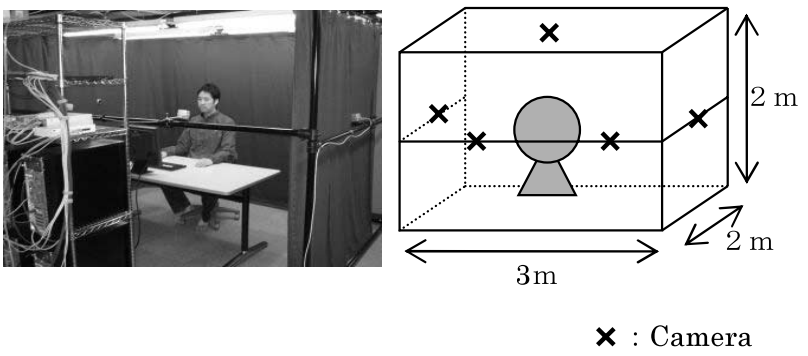

Fig. 3 Experimental Environment

The generated model in the modeling process is sent to the rendering PC via the network and displayed on the Perspecta. For sending the data, we have used the networking toolkit called QUANTA ${ }^{(16)}$.

\section{Experimental Environments and Results}

We built a studio for image capturing system as we can see in Fig. 3 for evaluation purposes. We used a set of calibrated digital cameras (Sony DFW-VL500) for capturing images in a size of $320 \times 240$ at 15 FPS. These images are sent to the PCs via IEEE1394 connection. We used a volume field resolution of $96 \times 96 \times 96$ and we measured the average computational time. The voxel size is $10 \mathrm{~mm}$. All PCs in this capturing system have $3.06 \mathrm{GHz}$ Intel Xeon processors, $2 \mathrm{~GB}$ of RAM and run Debian GNU/Linux. The rendering $\mathrm{PC}$ has $2.8 \mathrm{GHz}$ Intel Pentium 4 processor, $1 \mathrm{~GB}$ of RAM and run Windows2000. The average throughput of this system is 2.63 FPS. Some reconstruction results are shown in Fig. 4. In our system, objects are generated having the structure of the SVL, and each voxel has its own index number and RGB color information. Some results of displaying the transferred object onto Perspecta are shown in Fig. 5.

\section{Conclusions}

In this paper, we described a 3D modeling and displaying system for volume communication. Our proposed system is composed of two basic techniques. One is the
3D modeling technique. We build a studio for image capturing system consisting of a set of cameras and a PC cluster which runs the proposed parallel voxel coloring method in collaboration with the parallel volume intersection method. In our experiment, we can verify that the object in real-world is reconstructed in the PC at interactive frame rate. Another one is the $3 \mathrm{D}$ displaying technique using the omni-directional display. By transferring the reconstructed object to the rendering PC and displaying on Perspecta, it becomes possible to see a remote object in real-world from different viewpoints at the same time. As a future work, we expect to work on a more optimized algorithm for visibility determination and color assignment.

\section{Acknowledgements}

This work has been partly supported by the Japan Ministry of Education, Science, Sports and Culture's Grant-in-Aid for IT Program, 2004.

\section{References}

( 1 ) Chen, W.C., Towles, H., Nyland, L., Welch, G. and Fuchs, H., Toward a Compelling Sensation of Telepresence: Demonstrating a Portal to a Distant (Static) Office, Proc. of the 11th IEEE Visualization, (2000).

(2) Kauff, P. and Schreer, O., An Immersive 3D VideoConferencing System Using Shared Virtual Team User Environments, Proc. of the 4th Int. Conf. on Collaborative Virtual Environments, (2002).

(3) http://www.sharp.co.jp/products/pcrd13d/index.html (Japanese)

( 4 ) http://www.sanyo.co.jp/koho/hypertext4/0209newsj/0910-1.html (Japanese)

( 5 ) http://www.actuality-systems.com/

( 6 ) Seitz, S. and Dyer, C., View Morphing, Proc. of SIGGRAPH '96, (1996), pp.21-30.

( 7 ) Saito, H., Kimura, M., Yaguchi, S. and Inamoto, N., View Interpolation of Multiple Cameras Based on Projective Geometry, Proc. of Int. Workshop on Pattern Recognition and Understanding for Visual Information Media, (2002).

( 8 ) Wada, T., Wu, X., Tokai, S. and Matsuyama, T., Ho- 

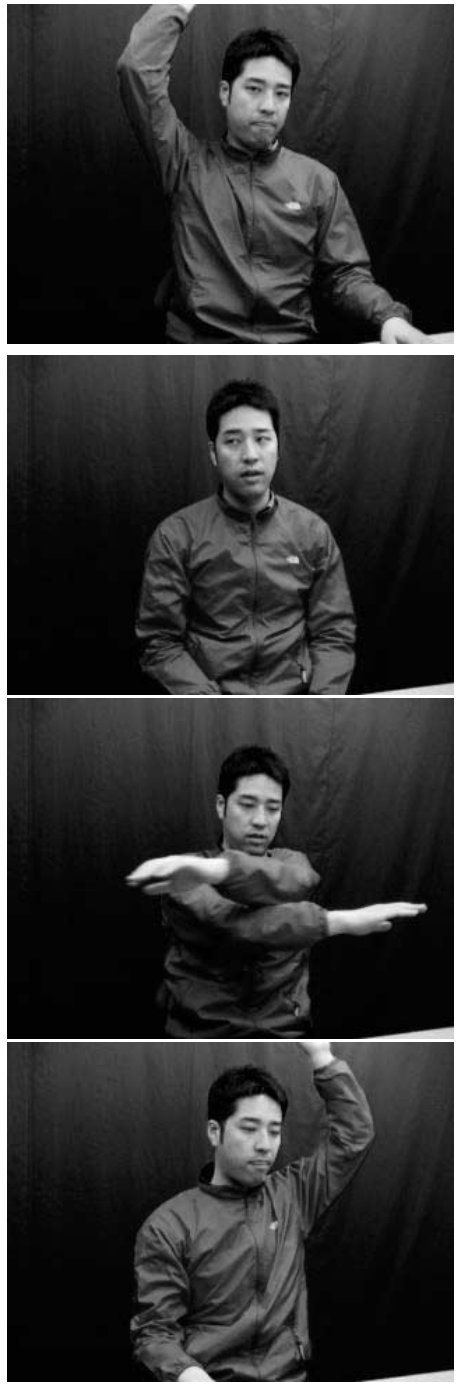

(a)
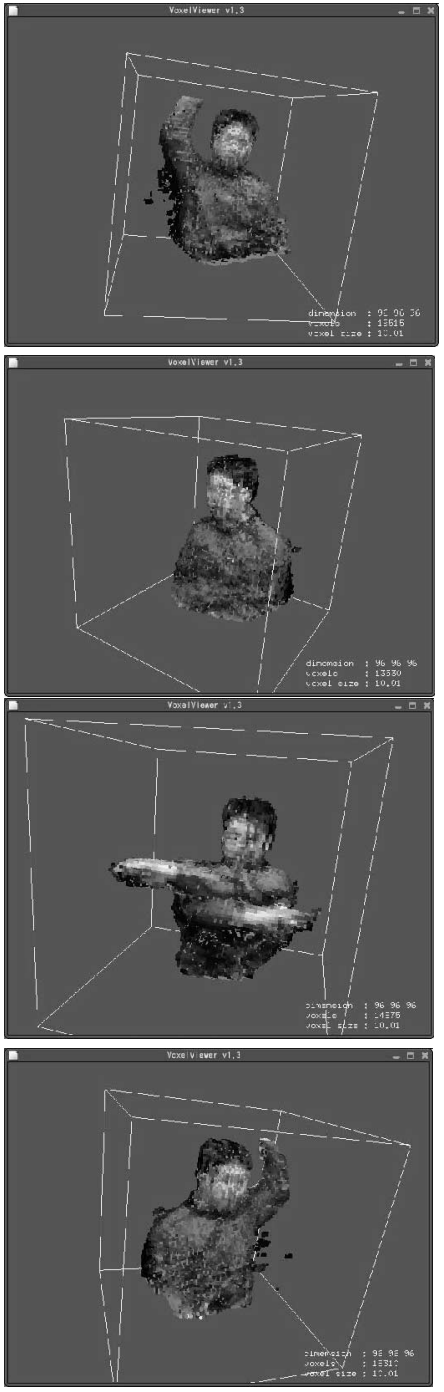

(b)
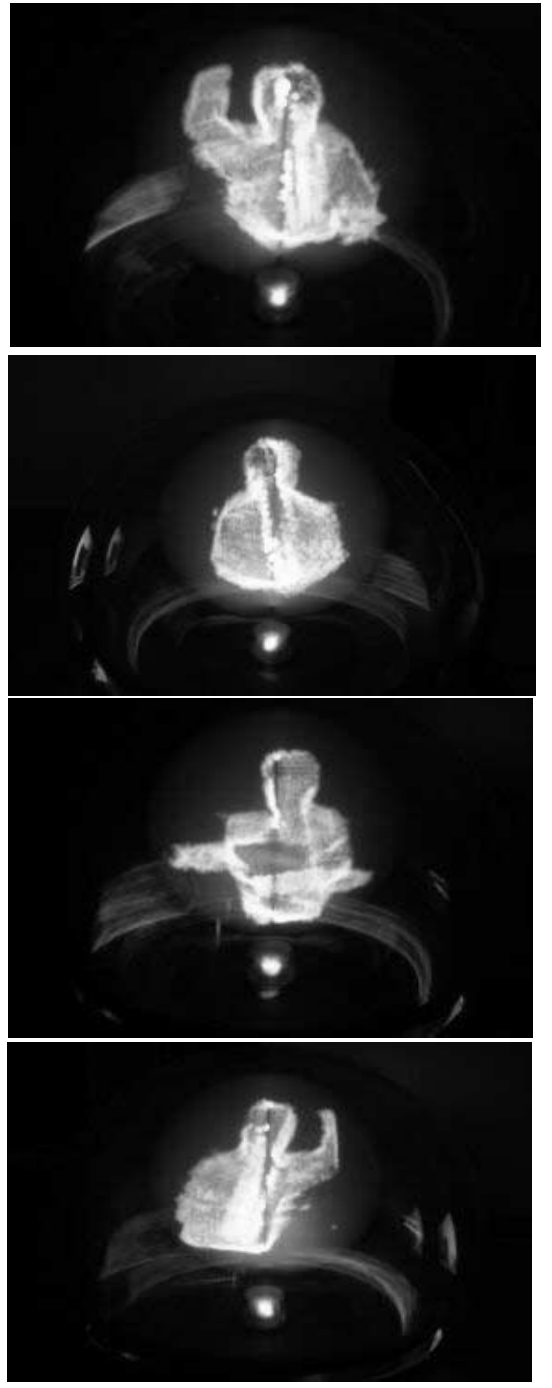

(c)

Fig. 5 (a) Captured images, (b) Reconstructed images, (c) Images on Perspecta

mography Based Parallel Volume Intersection: Toward Real-Time Volume Reconstruction Using Active Cameras, Proc. of Computer Architectures for Machine Perception, (2000), pp.331-339.

( 9 ) Li, M., Magnor, M. and Seidel, H., Hardware Accelerated Visual Hull Reconstruction and Rendering, Proc. of Graphics Interface, (2003), pp.65-72.

(10) Seitz, S. and Dyer, C., Photorealistic Scene Reconstruction by Voxel Coloring, Proc. of the IEEE Conf. on Computer Vision and Pattern Recognition, (1997), pp.1067-1073.

(11) Slabaugh, G., Culbertson, B. and Malzbender, T., A Survey of Methods for Volumetric Scene Reconstruction form Photograph, Int. Workshop on Volume Graphics 2001, (2001).
(12) Slabaugh, G., Culbertson, W., Malzbender, T., Stevens, M. and Schafer, R., Methods for Volumetric Reconstruction of Visual Scene, Int. Journal of Computer Vision, Vol.57, No.3 (2004), pp.179-199.

(13) Culbertson, W. and Malzbender, T., Generalized Voxel Coloring, Proc. of the Int. Workshop on Vision Algorithms: Theory and Practice, (1999), pp.100-115.

(14) Proc, A. and Dyer, C., Towards Real-Time Voxel Coloring, Proc. of Image Understanding Workshop, (1998), pp.315-321.

(15) Sainz, M., Bagherzadeh, N. and Susin, A., Hardware Accelerated Voxel Carving, Proc. of 1st IberoAmerican Symposium in Computer Graphics, (2002), pp.289-297.

(16) http://www.evl.uic.edu/cavern/quanta/ 